\title{
Clasificación de patologías presentes en la columna vertebral mediante técnicas de máquinas de aprendizaje
}

Classification of pathologies present in the spinal column through learning machinery techniques

Classificação de patologias presentes na coluna vertebral por meio de técnicas de aprendizado de máquinas

\section{Diego Fernando Ramírez-Jiménez ${ }^{1}$ Julián David Quintero-Ospina²}

Recibido: julio 16 del 2018

Aprobado: octubre 24 del 2018 Disponible en línea: enero 15 del 2019

Cómo citar este artículo:

D. F. Ramírez-Jiménez y J. D. Quintero Ospina, “Clasificación de patologías presentes en la columna vertebral mediante técnicas de máquinas de aprendizaje", Revista

Ingeniería Solidaria, vol. 25, n. ${ }^{\circ}$ 1, 2019. DOI: https://doi.org/10.16925/2357-6014.2019.01.05

Artículo de investigación. https://doi.org/10.16925/2357-6014.2019.01.05

1 Facultad de Ingeniería, Universidad del Valle, Cali, Colombia ORCID: https://orcid.org/0000-0001-9050-666X

Correo electrónico: ramirez.diego@correounivalle.edu.co

2 Facultad de Ingeniería, Universidad del Valle, Cali, Colombia ORCID: https://orcid.org/0000-0002-1761-2113 


\section{Resumen}

Introducción: el artículo es resultado de la investigación "Estudio de patologías presentes en la columna vertebral empleando técnicas de inteligencia artificial como apoyo a los procesos de diagnóstico", desarrollada en la Universidad del Valle entre 2016 y 2017.

Problema: con frecuencia, los estudios y análisis que a menudo se realizan a las afecciones de salud en seres humanos con frecuencia son invasivos, lo cual conlleva problemas mayores.

Objetivo: aportar un método de estudio a partir de los atributos biomecánicos de seres humanos para la detección de patologías que se presentan en la columna vertebral.

Metodología: el trabajo se fundamentó en probar tres técnicas de reconocimiento de patrones; Bayes como técnica clásica de reconocimiento; y técnicas inteligentes como las redes neuronales de base radial (RBF), máquinas de soporte vectorial (SVM) y redes neuronales probabilísticas (PNN).

Resultados: durante el proceso de clasificación de las patologías a tratar, la que mejores resultados aportó fue la técnica de PNN, mientras que las demás presentaron buenos resultados de clasificación para una patología en particular.

Conclusión: se comprobó que la aplicación de estas técnicas de estudio aporta características importantes a los procesos de diagnóstico de patologías presentes en la columna vertebral, tales como hernia discal y espondilolistesis.

Originalidad: este trabajo se realizó con información de pacientes reales, y presenta técnicas de estudio y resultados importantes sobre el diagnóstico de patologías de columna vertebral.

Limitaciones: el estudio de patologías de columna vertebral requiere tener más información sobre los atributos biomecánicos de los seres humanos.

Palabras clave: atributos biomecánicos; columna vertebral; funciones de base radial; máquinas de soporte vectorial; patologías, teorema de Bayes; redes neuronales probabilísticas.

\section{Abstract}

Introduction: This paper shows the result of research entitled "Study of pathologies present in vertebral column using artificial Intelligence Techniques as support of diagnostic processes", developed in University of Valle between the years 2016 and 2017.

Problem: Studies and analyzes that are carried out on the health conditions of human beings are often invasive, which leads to greater issues.

Objective: To provide a method of study from biomechanical attributes of human beings for the detection of pathologies present in vertebral column.

Methodology: The study was based on testing three pattern recognition techniques, Bayes as a classic recognition technique, and intelligent techniques such as Radial Basis Functions Neural Networks (RBF), Support Vector Machines (SVM) and Probabilistic Neural Networks (PNN).

Results: During the classification process of the pathologies to study, the best results were obtained using PNN, while the other ones presented good classification results for a particular pathology.

Conclusion: It was proven that study techniques contributes important characteristics to diagnosis processes of pathologies present in the vertebral column, such as disk hernia and spondylolisthesis.

Originality: This study was carried out with information from real patients, providing study techniques and important results on the diagnosis of vertebral column pathologies. 
Limitations: The study of vertebral column pathologies requires more information about the biomechanical attributes of human beings.

\section{Keywords:}

\section{Resumo}

Introdução: o artigo é resultado da pesquisa "Estudo de patologias presentes na coluna vertebral empregando técnicas de inteligência artificial como apoio aos processos de diagnóstico", desenvolvida na Universidad del Valle, entre 2016 e 2017.

Problema: os estudos e análises que costumam ser realizados sobre as condições de saúde em seres humanos são frequentemente invasivos, o que implica problemas maiores.

Objetivo: oferecer um método de estudo a partir dos atributos biomecânicos de seres humanos para detectar patologias que se apresentam na coluna vertebral.

Metodologia: o trabalho é fundamentado em testar três técnicas de reconhecimento de padrões; Bayes como técnica clássica de reconhecimento; e técnicas inteligentes como as redes neurais de base radial (RBF), máquinas de vetores de suporte (SVM) e redes neurais probabilísticas (PNN).

Resultados: durante o processo de classificação das patologias a serem tratadas, a técnica que apresentou os melhores resultados foi a PNN, enquanto as demais apresentaram bons resultados de classificação para uma patologia em particular.

Conclusão: comprovou-se que a aplicação dessas técnicas de estudo oferece características importantes aos processos de diagnóstico de patologias presentes na coluna vertebral, tais como hérnia de disco e espondilolistese.

Originalidade: este trabalho foi realizado com informação de pacientes reais e apresenta técnicas de estudo e resultados importantes sobre o diagnóstico de patologias de coluna vertebral.

Limitações: o estudo de patologias de coluna vertebral requer mais informação sobre os atributos biomecânicos dos seres humanos.

Palavras-chave: atributos biomecânicos; coluna vertebral; funções de base radial; máquinas de vetores de suporte; patologias, teorema de Bayes; redes neurais probabilísticas.

\section{Introducción}

El mundo actual se encuentra en un proceso de cambio acelerado, en virtud de los múltiples avances tecnológicos que se presentan a diario. Estos interfieren de forma directa o indirecta en el entorno y traen consigo cambios significativos en hogares, medios de transporte, oficinas, industrias, y, sobre todo, en la medicina. Por lo anterior, es de gran importancia explorar posibles nuevas técnicas y tecnologías de implementación en la detección de patologías que se presentan en el ser humano, a fin de prevenir enfermedades a futuro.

Los algoritmos computacionales y técnicas de máquinas de aprendizaje (MLT) ostenta propiedades que les permiten brindar soluciones eficientes a problemas de detección de patologías en la columna vertebral, ya que se trata de un procedimiento 
no invasivo en el cual se realiza una clasificación de parámetros ajustable a sistemas con múltiples clases, que pueden ser o no lineales [1]. Una muestra de la capacidad que tienen las máquinas de aprendizaje para procesos de clasificación está en la técnica de máquinas de soporte vectorial: a partir de hiperplanos se pueden establecer fronteras de separación entre las diferentes clases de datos.

La capacidad que tienen las máquinas de aprendizaje de adaptarse casi a cualquier tipo de problema de estudio ha permitido su uso en diferentes campos de acción, dentro de los cuales se destacan, de un lado, la medicina, con aplicaciones para estudios de cardiografía (señales ECG), gastroenterología, neumología, radiología, neurología (señales EEG), electromiografía (señales EMG); y de otro, la biología, con aplicaciones para el reconocimiento y clasificación de especies [1], [2].

Por lo anterior, y con el fin de establecer otras alternativas para el diagnóstico clínico convencional, el cual suele ser en algunos casos costoso, demorado y poco eficiente, el desarrollo de este trabajo buscó contribuir al desarrollo de posibles métodos de diagnóstico no invasivos para la identificación de patologías presentes en columna vertebral desde la academia: a partir de un conjunto de muestras y datos tomados a cada individuo para los atributos que hacen parte de la morfología pélvica, tales métodos permitirían extraer características específicas que, al compararse con datos reales que describan el comportamiento de un individuo normal, llevarían a determinar si existe alguna patología en los individuos estudiados y su grado de afección. Para lograr esto, se dispuso un conjunto de 310 datos diferentes que debían separarse por clases; es decir, identificar los subconjuntos de datos de individuos que padecían o no algún tipo de patología. Posteriormente, se realizó un procedimiento para extraer las características más relevantes que permitieran determinar alguna patología presentada - en este caso, las condiciones que tienen los atributos biomecánicos de la morfología pélvica-. Por último, se aplicaron algunas técnicas de clasificación y reconocimiento de patrones (como las mencionadas en la sección 2) para realizar los procesos de entrenamiento y validación a partir de los cuales se pudieran obtener los resultados finales para los diferentes clasificadores.

Este artículo se encuentra organizado en cinco secciones, incluida la presente introducción. La segunda sección contiene una descripción de antecedentes teóricos; la tercera comprende una breve explicación de los fundamentos teóricos y las técnicas de clasificación implementadas; en la tercera se presentan la discusión y los resultados obtenidos con el uso de las técnicas de clasificación implementadas en el conjunto de datos de patologías de la columna vertebral; y la cuarta comprende las conclusiones del trabajo realizado. 


\subsection{Antecedentes}

Uno de los rasgos más importantes de los seres humanos radica en la capacidad de aprender. Estos procesos de aprendizaje pueden ser muy distintos de unos individuos a otros, e incluso el perfeccionamiento de lo aprendido puede hacerse de forma y en tiempos diferentes. De forma similar se da el aprendizaje de máquina -conocido como Machine Learning ( $\mathrm{ML}$ ) - , en el que la idea general consiste en estudiar una serie de datos que pueden dividirse en tres etapas: preprocesamiento, extracción de características y clasificación. Estas pueden obtenerse a partir de diferentes métodos o técnicas de clasificación y reconocimiento, de forma independiente o no, o bien basadas en procesos estadísticos, los cuales se convierten en inferencias a partir de descripciones generales [3], [4]. Los procesos de aprendizaje se basan en entrenamientos que se realizan con los datos o muestras que se tienen de cualquier sistema, en los que el proceso final de clasificación consiste en un análisis discriminante de acuerdo con las características de los patrones con los cuales se desee compararlos.

En ingeniería se pueden encontrar diferentes enfoques respecto de los procesos en los cuales se aplican técnicas de máquinas de aprendizaje y reconocimiento de patrones: por ejemplo, Duda [4] establece que los enfoques de este tipo de técnicas son no paramétricas y mucho más empíricas. Otros enfoques dan lugar a sus orígenes en el aprendizaje automático [5], la inteligencia artificial [6], las redes neuronales artificiales [3] y la minería de datos [7].

Desde sus inicios, en la década de 1950, la inteligencia artificial se ha implementado año tras año en una gran cantidad de campos de acción, entre los cuales se destacan las ciencias de la salud debido a los múltiples avances tecnológicos alcanzados en años recientes en materias de técnicas de estudio, diagnóstico y procesos de intervención hacia los pacientes. Lo anterior configura un estado del arte de enormes proporciones, de las cuales se citarán aquí los más relevantes en lo que atañe al estudio de patologías de la columna vertebral, así como sus métodos y técnicas de clasificación.

Las máquinas de aprendizaje tienen sus inicios en la década de 1950, época en la que se las consideraba más cercanas a la fantasía que a la realidad. En un principio, el desarrollo de los sistemas de aprendizaje autónomo estuvo liderado por teóricos importantes de la época como Thomas Bayes, Adrien-Marie Legendre y Pierre-Simon Laplace, pero fue en 1950 cuando Alan Turing desarrolló el test que lleva su apellido: consistía en un juego en el que una máquina se hacía pasar por un humano a través de una conversación con uno de ellos. Dos años más tarde, Arthur Samuel desarrolló una máquina para jugar damas que tenía la capacidad de aprender y mejorar a través de cada juego. Finalmente, la revolución en los sistemas de máquinas de aprendizaje 
se dio en 1957, cuando Frank Rosenblatt desarrolló con el perceptrón una red neuronal artificial (ANN), que se convirtió en el primer algoritmo que tenía la capacidad de aprender a aprender [8].

En el ámbito académico se han desarrollado diferentes trabajos enfocados a la aplicación de técnicas de máquinas de aprendizaje para la clasificación y el diagnóstico de patologías en columna vertebral. Entre estos se encuentra "Application of J48 and Bagging for Classification of Vertebral Column Pathologies", cuyo objetivo principal radicó en establecer un sistema que pudiese ayudar a los radiólogos de los hospitales en Indonesia a realizar diagnósticos precisos de las patologías que se presentan en la columna vertebral. No obstante, debido a la falta de información real de pacientes que padecieran enfermedades como hernia de disco y espondilolistesis, dicho estudio se desarrolló con información obtenida de una base de datos real de la ucı y se aplicaron las técnicas de clasificación de árbol de decisión (J48) y Bagging [9].

En Islamabad (Pakistán) existe un proyecto de investigadores locales que sirve como ejemplo de la aplicación de estos proyectos en el área de la medicina y el diagnóstico de patologías presentes en la columna vertebral; Ansari et al. afirman al respecto que un sistema o elemento de clasificación inteligente y sofisticado puede ser de gran utilidad para tomar decisiones en áreas de estudio donde los médicos especialistas no están presentes [10]. El trabajo consistió, entonces, en recopilar los datos de los pacientes a través de imágenes de resonancia magnética (MRI); se utilizaron técnicas de clasificación como la red neuronal de propagación, la red neuronal de regresión generalizada y las máquinas de soporte vectorial [10].

De forma paralela con lo anterior, investigadores de las universidades de Amasya y Selcuk (Turquía) realizaron una publicación sobre el diagnóstico de patologías en columna vertebral a través de técnicas como perceptrón multicapa (MLP) y teorema de Bayes. El trabajo realizado consistió en presentar un sistema de diagnóstico asistido por computadora (CAD) que pudiera ayudar minimizar los errores humanos en el diagnóstico [11].

\section{Metodología}

\subsection{Material}

El conjunto de datos utilizado para el diagnóstico de personas con problemas de columna vertebral fue dispuesto por el Centro de Máquinas de Aprendizaje y Sistemas Inteligentes de la Universidad de California en Irvine para consultarse en línea [13]; está organizado en dos grupos de clasificación diferentes pero relacionados de forma 
directa, como se observa en la tabla 1. Así entonces, el grupo 1 se divide en individuos que pertenecen a tres categorías - normal, hernia discal y espondilolistesis-, mientras que el 2 cuenta con la misma cantidad de individuos, pero divididos en dos categorías: normal y anormal (incluye hernia discal y espondilolistesis) [12], [13].

Tabla 1. Clasificación del conjunto de datos por grupos

\begin{tabular}{ccccc}
\hline \multirow{2}{*}{ Grupos } & \multicolumn{3}{c}{ Categorías } & Total de pacientes \\
\cline { 2 - 4 } & Normal & Hernia discal & Espondilolistesis & \\
\hline Grupo 1 & 100 & 60 & 150 & 310 \\
\hline Grupo 2 & 100 & 210 & & 310 \\
\hline
\end{tabular}

Fuente: elaboración propia

De la información de muestras en la tabla 1 se evidencia que el $49 \%$ de los datos corresponde a individuos que padecen espondilolistesis; $32 \%$ a individuos que no padecen ninguna patología (normal); y $19 \%$ a personas con hernia discal [13]. Sin embargo, la correspondencia de estas muestras respecto a la ocurrencia real en una consulta cambia de forma significativa, pues existen múltiples patologías de columna que se presentan comúnmente en los individuos -las cuales pueden ser congénitas o adquiridas - [14]. Sin embargo, fuentes indican que aproximadamente el $6 \%$ de los hombres y el $3 \%$ de las mujeres padecen espondilolistesis, ya que es una patología que se desarrolla principalmente en personas que realizan grandes esfuerzos físicos, tales como deportistas de alto rendimiento; mientras que la patología de hernia discal corresponde al 3 \% de los dolores de espalda [15]. Con base en esta información, puede usarse un número diferente de muestras para ajustar el conjunto de datos a la probabilidad de ocurrencia real en una consulta, y luego proseguir a la clasificación. Con esto, para cada uno de los registros del conjunto de datos se dispone de seis atributos biomecánicos, derivados de la orientación de la pelvis y la espina lumbar (véase la figura 1a).

La incidencia pélvica (PI) puede definirse como el ángulo subtendido por la línea $\overline{\mathrm{o}}$, que es trazada desde el centro de la cabeza femoral hasta el punto medio de la placa terminal del sacro y una línea perpendicular al centro de la placa terminal del sacro. La placa terminal del sacro es definida por el segmento de línea bc. La pendiente del sacro (ss) es definida por el ángulo compuesto por la línea horizontal de referencia (HRL) y la línea $\overline{b c}$. La inclinación de la pelvis (PT), por su parte, se define por el ángulo comprendido por una línea de referencia vertical (VRL) originado desde el centro de la cabeza femoral (o) y el radio pélvico $\overline{\mathrm{oa}}$; y el grado de espondilolistesis corresponde 
al porcentaje de desplazamiento entre el plato inferior de la quinta vértebra lumbar y el sacro [2], [16].

Por otra parte, la espondilolistesis puede ser identificada a partir del grado de PI (véase la figura 1b). En la imagen de la izquierda se observa que el ángulo está dentro de los valores normales, en la imagen del centro se puede ver una postura normal, aunque el ángulo de PI supera el valor máximo permitido y finalmente, en la imagen de la derecha, se observa un individuo con espondilolistesis.

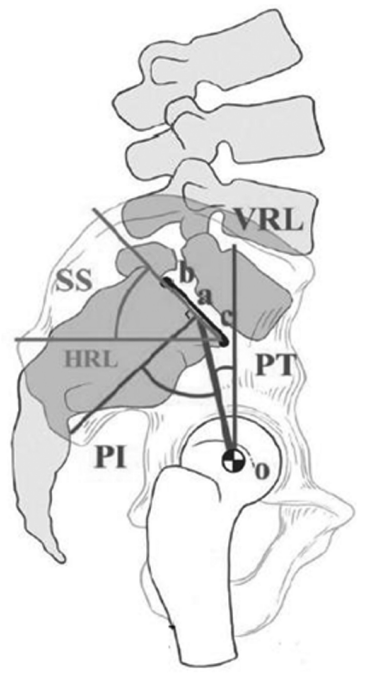

a

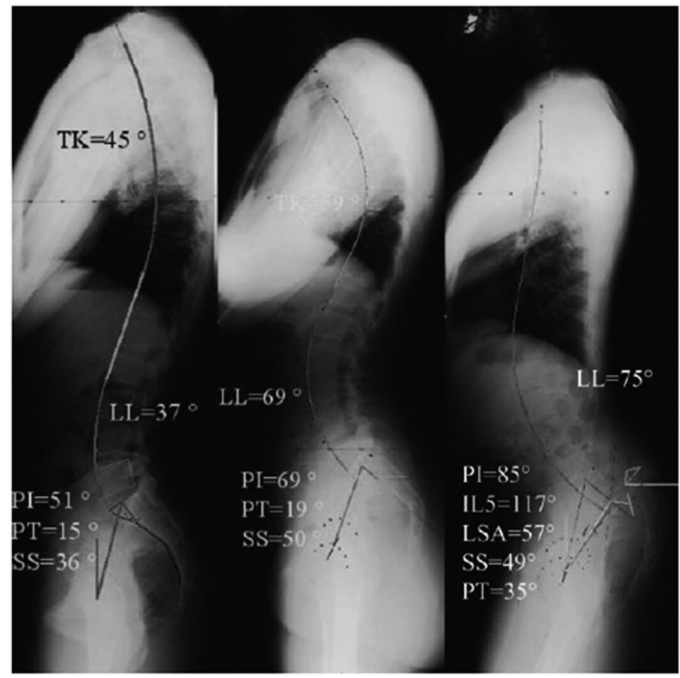

b

Figura 1. a) Atributos biomecánicos de la columna vertebral de un individuo.

En esta se pueden apreciar los ángulos y pendientes que forman la pelvis y el sacro respecto a la cabeza femoral en una persona normal. b) Representación de dos pacientes que se encuentran en condición normal y uno que padece espondilolistesis, a través de una toma de rayos $X$

Fuente: Abdrabou [17]

\subsection{Método}

Para el estudio que se describe aquí se empleó una metodología una metodología en la que se combinaron cuatro algoritmos para la clasificación de patologías de la columna vertebral: redes neuronales probabilísticas (PNN), máquinas de soporte vectorial (SVM), clasificador Naïve Bayes y redes neuronales con función de base radial (RBF) (figura 2). 


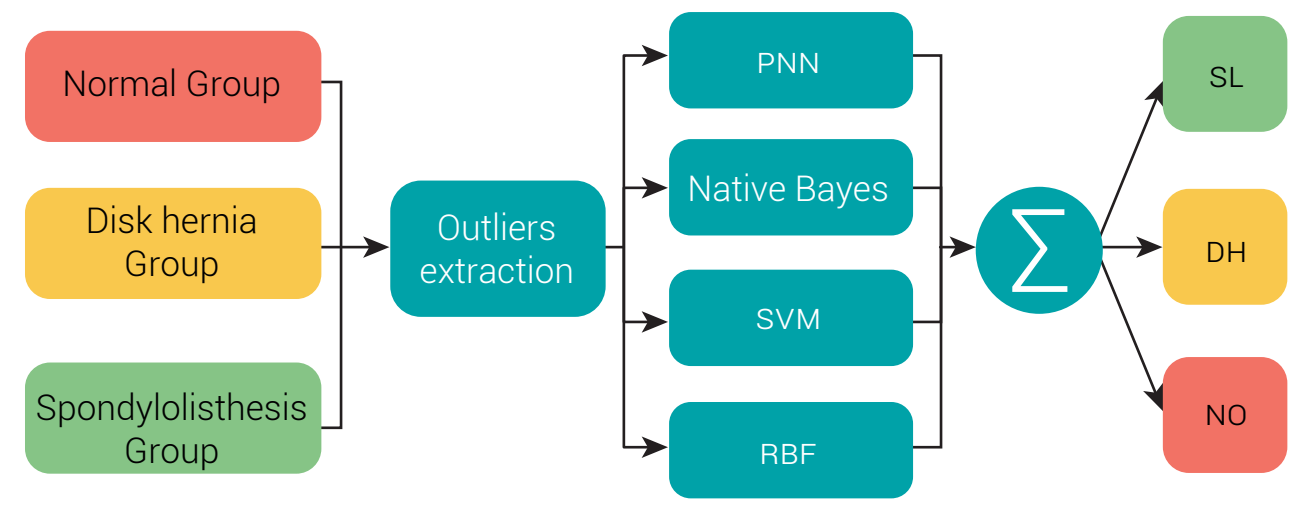

Figura 2. Diagrama de la metodología de clasificación propuesta.

Se tenían los datos, a los cuales se les aplicó una extracción de outliers para

luego introducirlos en los clasificadores. Finalmente, se obtuvieron los resultados de acuerdo con el entrenamiento y supervisión para cada patología

Fuente: elaboración propia

\subsubsection{Supresión de outliers}

Una primera exploración del conjunto de datos para el diagnóstico de personas con problemas de columna vertebral muestra el alto grado de superposición que existe entre las tres clases, siendo el grado de superposición más elevado entre los datos normales y de hernia discal (figura 3).

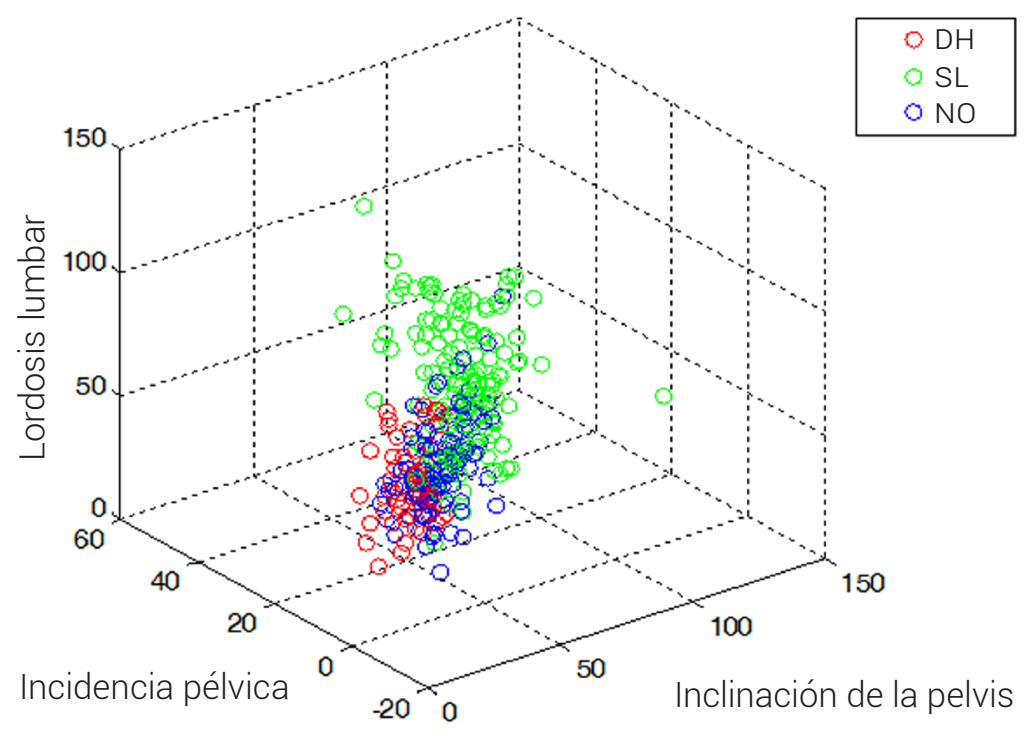

Figura 3. Gráfico de dispersión de los atributos PI, PT y lordosis lumbar.

Una observación importante es la existencia de outliers en las tres clases, un hecho evidente por el número de puntos de una clase dada que se encuentran muy alejados del centro de la clase respectiva

Fuente: elaboración propia 
Los outliers aparecen en la mayoría de los casos cuando se trata de muestras que contienen ruido, que pueden acarrear errores de clasificación debido a su baja frecuencia de ocurrencia. En consecuencia, para evitar confusiones en los clasificadores y mejorar su tasa de acierto, es recomendable llevar a cabo procesos de extracción de outliers. En el caso particular de este trabajo, la detección de estos últimos se realizó mediante el cálculo de la distancia de Mahalanobis [18], que describe la distancia entre cada punto de datos y el centroide. Cuando un punto de datos se encuentra en el centroide, la distancia es cero; y cuando se encuentra distante de este, la distancia es mayor que cero. Por lo tanto, los puntos de datos que se encuentran lejos del centroide se consideran valores atípicos. Mediante este enfoque, los outliers pueden identificarse en un espacio multidimensional para ser eliminados después (figura 4).

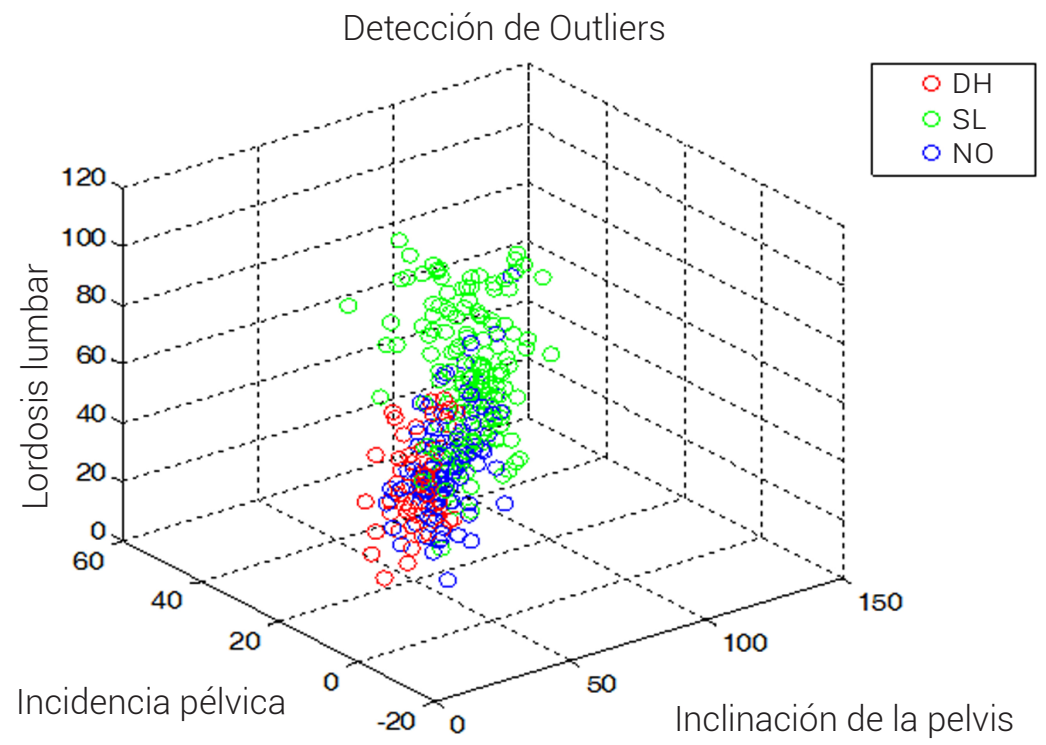

Figura 4. Gráfico de dispersión de los atributos de PI, PT y lordosis lumbar sin outliers. El proceso de separación se hace teniendo en cuenta las distancia entre los diferentes puntos de cada clase y el centroide

Fuente: elaboración propia

Dado lo anterior, se considera que un dato x es un outlier si satisface la siguiente condición:

$$
K<\sqrt{\left(x-\mu_{i}\right)^{\top} C_{i}^{-1}\left(x-\mu_{i}\right)}
$$

Donde K es el valor crítico de la máxima distancia de Mahalanobis al cuadrado, la cual se estima con base en la distribución Fisher con $n$ grados de libertad y un nivel de significancia a, a los cuales se les asignó valores de 6 y 0,1, respectivamente [19]. 


\subsubsection{Algoritmos de clasificación}

El primer algoritmo de clasificación escogido fue SVM. Estos son algoritmos de clasificación estadísticos usados para la clasificación de problemas en los cuales se tienen distintas clases [12]. El proceso de aprendizaje consiste en encontrar el hiperplano $\mathrm{H}$ (véase la figura 5) a través de funciones que pueden ser lineales, no lineales, polinomiales o $\operatorname{RBF}[12,20,21]$.

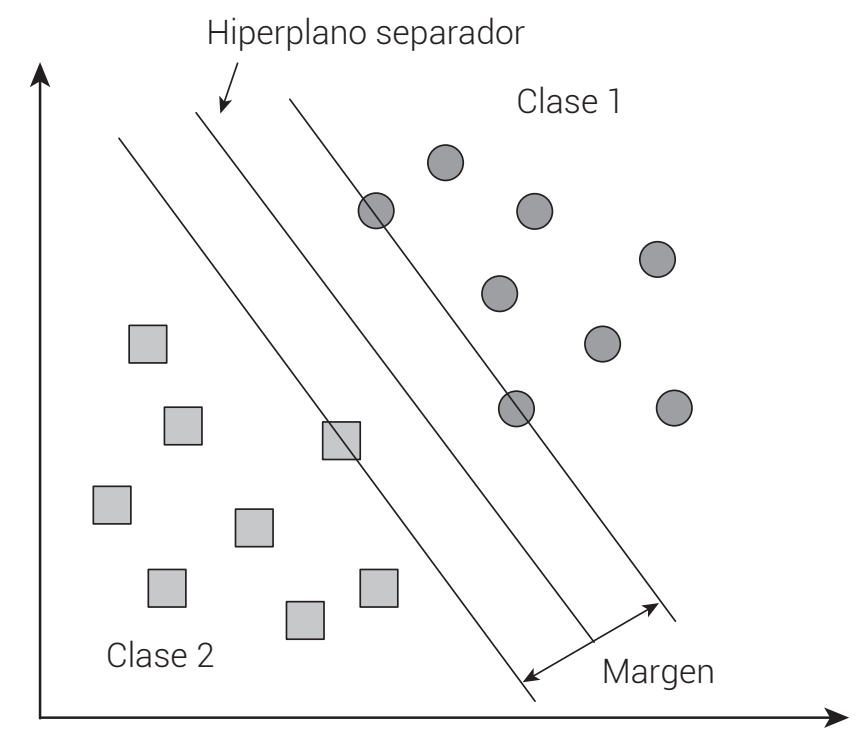

Figura 5. Separación de datos a través de máquinas de soporte vectorial (SVM) Fuente: Gutiérrez [16]

Para la clasificación de la información del conjunto de datos se tuvo en cuenta la función Kernel RBF, debido al desempeño y eficiencia que ostenta en el proceso de clasificación.

Por otro lado, los algoritmos RBF suelen usarse para la interpolación de datos multivariantes. Los primeros estudios y aplicaciones de este método se atribuyen a Powell (1987), y en la actualidad tienen gran aceptación en los sistemas de aprendizaje, debido a que asocian un sistema de ecuaciones que puede ser invertible incluso si la distribución de los puntos no presenta regularidad [22].

$$
y_{k}=\sum_{i} w_{k j} i_{j}+\theta_{k}=\sum_{i} w_{k j} \phi\left(r_{j}\right)+\theta_{k}
$$


Donde,

$$
\phi(r)=e^{-r^{2} / 2 \sigma^{2}}
$$

A simple vista, la arquitectura de una red RBF (véase la figura 6) no presenta diferencias significativas con otros sistemas de máquinas de aprendizaje, tales como las redes neuronales convencionales o el perceptrón multicapa (MLP) con aprendizaje supervisado, en los que la información fluye desde la capa de entrada hacia la salida de manera unidireccional.

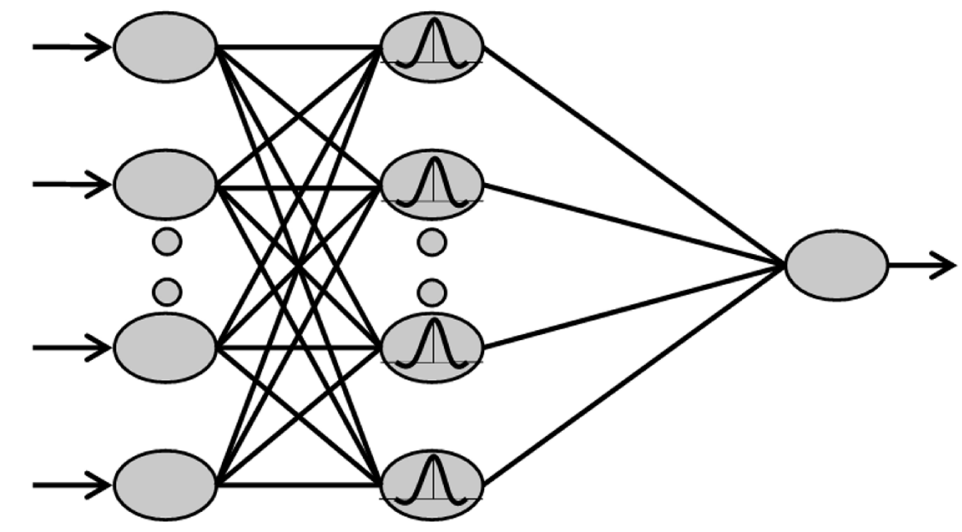

Figura 6. Arquitectura de una red RBF. La diferencia esencial entre esta y la del MLP radica en la capa oculta, cuya función de activación es centrada y del tipo base radial, normalmente gaussiana (ecuación 2). Por esta razón, los parámetros que caracterizan a esta capa oculta son el centro de la función de activación (centroide) y su respectiva desviación estándar

Fuente: elaboración propia

El teorema de Bayes, por su parte, es una técnica probabilística usada principalmente cuando se tienen entradas multidimensionales, que proporciona la distribución de probabilidad entre dos eventos (véase la figura 7). La probabilidad a posteriori de un sistema multidimensional con $C$ clases puede calcularse como:

$$
P\left(C_{k} \mid x\right)=\frac{p\left(x \mid C_{k}\right) P\left(C_{k}\right)}{p(x)}
$$

Donde

$$
p(x)=\sum_{k=1}^{c} p\left(x \mid C_{k}\right) P\left(C_{k}\right) \text { y } \sum_{k=1}^{c} P\left(C_{k} \mid x\right)=1
$$


A pesar de su simplicidad, el teorema de Bayes puede sobrepasar en algunos casos a los métodos de clasificación más usados y avanzados [23].

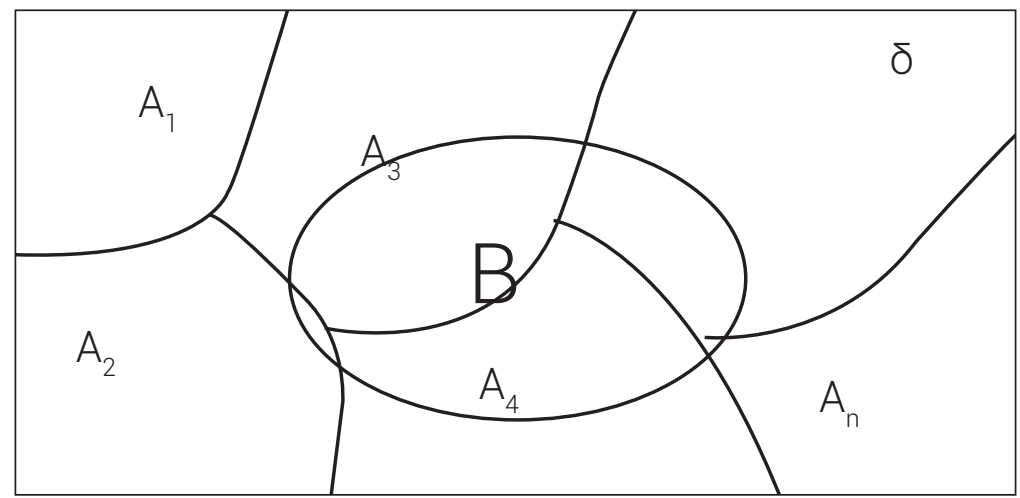

Figura 7. Espacio muestral $\delta$ formado por los eventos "A" $\mathrm{y}$ "B" Fuente: Babalik, Babaoğlu y Özkış [12]

Las PNN, al igual que otras técnicas que incluyen la implementación de técnicas de máquinas de aprendizaje como MLP, pueden emplearse para resolver problemas de clasificación de datos o especies. Una PNN (véase la figura 8) está compuesta por tres capas: de entrada, competitiva y de salida [19, 24].

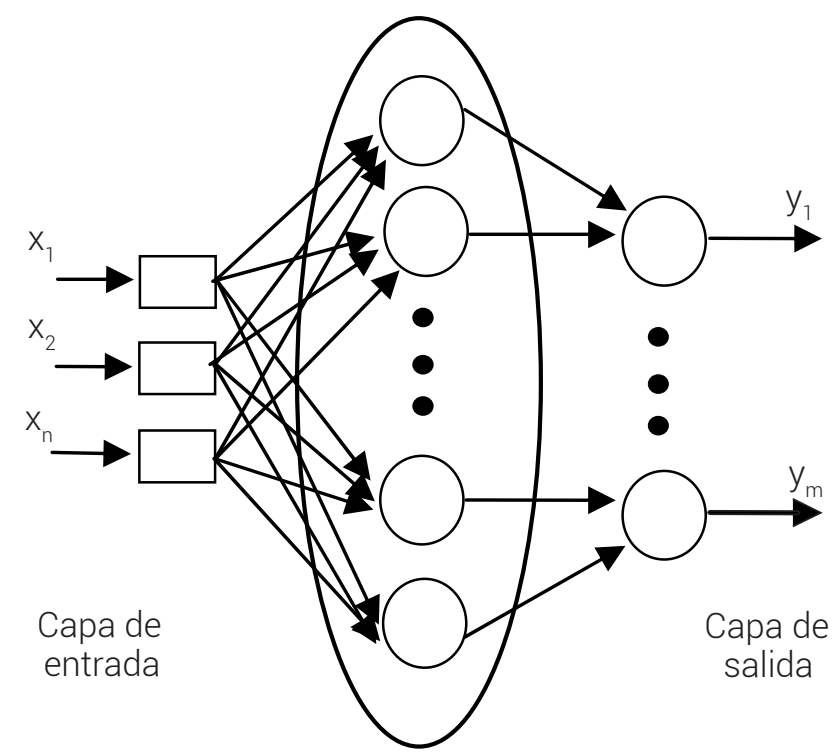

Capa

competitiva

Figura 8. Arquitectura de una PNN

Fuente: J. A. Cadena, C. J. M. Cadena y Pérez Londoño [24] 
A diferencia de otras técnicas, en las que las redes neuronales usan pesos para realizar los ajustes de acuerdo con los errores que se pueden presentar, el proceso de clasificación con una PNN no utiliza pesos: se hace a través de la comparación de patrones y cálculos de distancia entre la dispersión de los datos de tal forma que, cuando se presentan entradas a la red, la primera capa se encarga de obtener las distancias hacia los vectores de entrada de entrenamiento, produciendo un nuevo vector que indica el grado de cercanía entre la entrada presente en la red y la de entrenamiento. En la segunda capa se suman todas las contribuciones de las entradas para producir un vector de salida con las probabilidades obtenidas; y en la capa de salida se obtiene el valor de la probabilidad máxima [8] entregado por la función de la capa competitiva [19].

\subsubsection{Combinación de clasificadores}

Existen diversos métodos para obtener una salida mediante la combinación de clasificadores [25]. Para este caso se utilizó una combinación por promedio ponderado. Este se compone de un conjunto de redes entrenadas (es decir, expertos) que comparten una entrada común $x(\mathrm{p})$ para el patrón de entrenamiento p, y cuyas salidas individuales yi(p) se combinan mediante un promedio ponderado para producir una salida global $\mathrm{y}(\mathrm{p})$; es decir, la combinación se producirá según el peso o capacidad de clasificación que tenga cada uno de los algoritmos descritos en la etapa 2 para determinar la salida del método propuesto.

\section{Resultados}

En esta sección se presentan los resultados que se obtuvieron para los algoritmos de clasificación empleados, descritos en la sección anterior. En primera instancia, se presentan los resultados para los clasificadores implementados de forma independiente; es decir, se realizó el proceso de separación y clasificación de los elementos del conjunto de datos para las patologías estudiadas respecto de cada técnica empleada. Posteriormente, se presentan los resultados obtenidos al realizar la clasificación de las patologías tratadas, usando la combinación de los diferentes clasificadores que inicialmente se emplearon de forma individual.

\subsection{Clasificadores individuales}

Luego de eliminar los outliers presentes en las muestras de datos para cada clase, se implementaron los clasificadores de forma independiente: en estos se usó el $60 \%$ 
de los datos para realizar el entrenamiento y el $40 \%$ restante para el proceso de validación.

Primero se realizó la implementación de la red neuronal probabilística, cuya capa de entrada consta de seis atributos: ángulo de PI, PT, ángulo de lordosis lumbar, pendiente sacral, radio de la pelvis y grado de espondilolistesis. En la capa competitiva se usó una función de base radial con 182 neuronas (una neurona por dato); y en la capa de salida se obtuvieron los datos correspondientes a dos patologías (hernia discal y espondilolistesis) y el estado normal.

Los resultados obtenidos, que se muestran en la tabla 2, se obtuvieron a partir de una relación cruzada (leave-one-out) con todos los datos disponibles para realizar tanto los procesos de entrenamiento y validación. El porcentaje de clasificación para la técnica de PNN (véase la figura 9) fue superior al $90 \%$; mientras que con SVM (véase la figura 10), Bayes (véase la figura 11) y RBF (véase la figura 12) se obtuvieron porcentajes de clasificación que oscilaron entre el 82 y 88 \%, siendo la técnica SVM la que menor rendimiento obtuvo. Finalmente, se realizó la clasificación a través de un modelo hibrido (véase la figura 12) entre los clasificadores usados, con lo que se alcanzó el mismo desempeño de la técnica de PNN.

Tabla 2. Resultados de clasificación

\begin{tabular}{cc}
\hline Técnica usada & Clasificación (\%) \\
\hline PNN & 90,2 \\
\hline RBF & 87,7 \\
\hline SVM & 82,8 \\
\hline Bayes & 86,1 \\
\hline Combinación de clasificadores & 90,2 \\
\hline
\end{tabular}

Fuente: elaboración propia

La figura 9 presenta la matriz de confusión generada para el clasificador con PNN. Cabe destacar que, en la matriz de confusión, 1 corresponde a la clase de hernia discal; 2, a espondilolistesis; y 3, a normal. De allí se puede observar la ocurrencia de cuatro falsos positivos (tres individuos normales, dos de los cuales fueron considerados por el sistema como con hernia discal y uno con espondilolistesis) y siete falsos negativos ( 6 individuos con hernia discal y uno con espondilolistesis) categorizados como normales. También se observa que existe una clasificación incorrecta entre patologías: el caso de un individuo con espondilolistesis fue categorizado por el clasificador como hernia discal. 


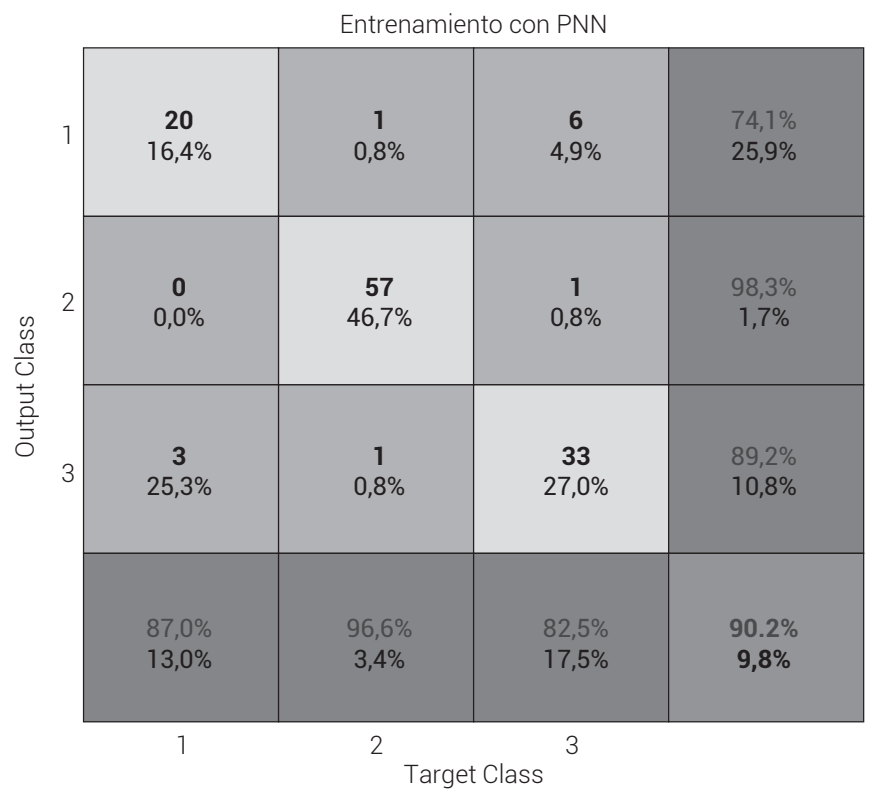

Figura 9. Resultados obtenidos con PNN

Fuente: elaboración propia

A partir del análisis de la matriz de confusión obtenida mediante un clasificador SVM (véase la figura 10), se puede observar la ocurrencia de 2 falsos positivos, 17 falsos negativos y 2 errores de clasificación entre patologías.

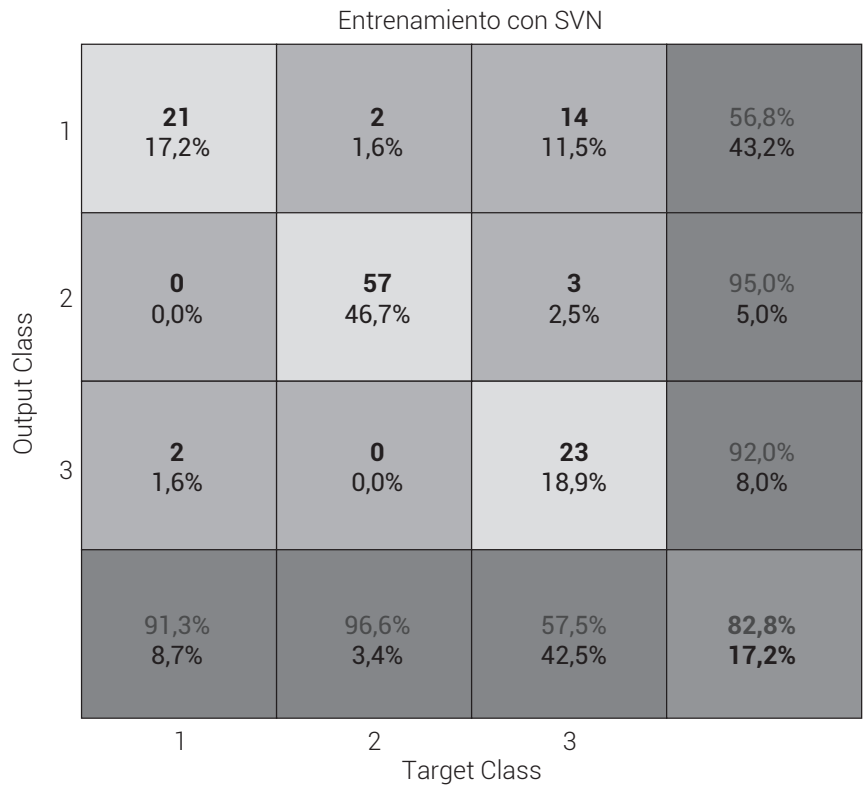

Figura 10. Resultados obtenidos con SVM

Fuente: elaboración propia 
En la figura 11 se muestra la matriz de confusión a partir de un clasificador con Naïve Bayes. Se puede observar la ocurrencia de 3 falsos positivos y 14 falsos negativos. Resulta importante enfatizar que este clasificador no presenta errores en la clasificación entre patologías.

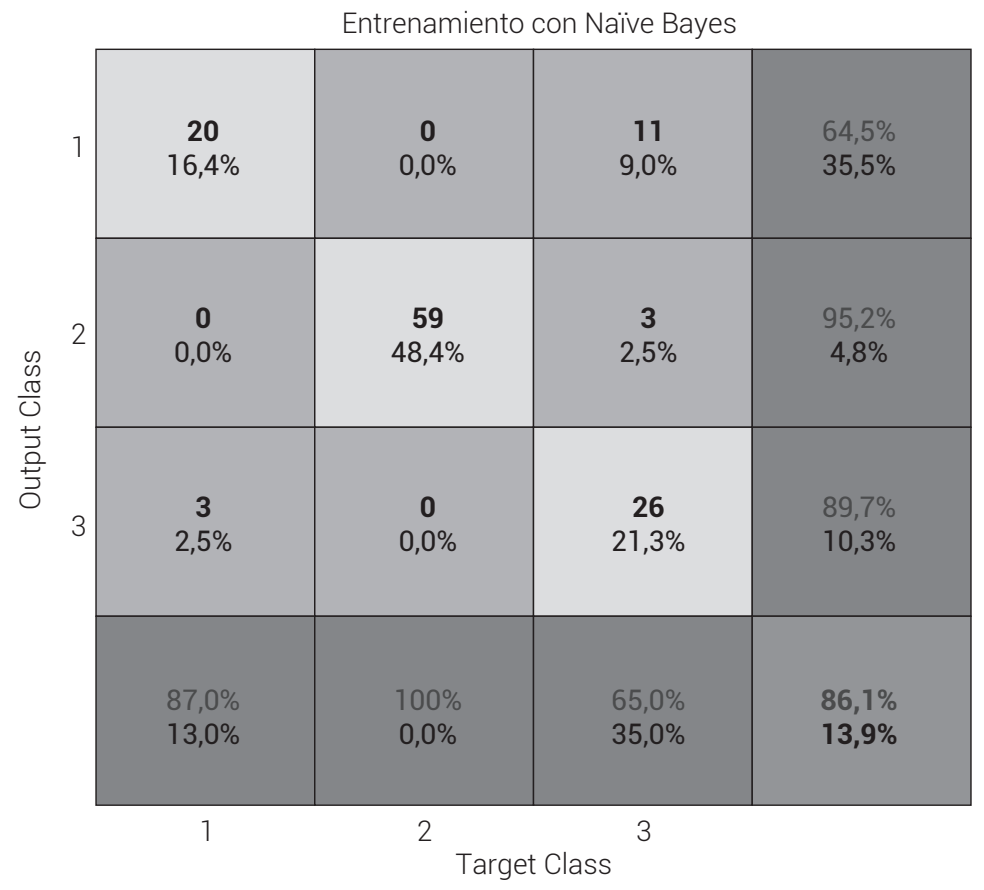

Figura 11. Resultados obtenidos con Naïve Bayes

Fuente: elaboración propia

En la figura 12 se presenta la matriz de confusión generada para el clasificador RBF, que presenta 5 falsos positivos y 10 falsos negativos. Al igual que el clasificador Naïve Bayes, RBF no muestra clasificaciones incorrectas entre patologías. 


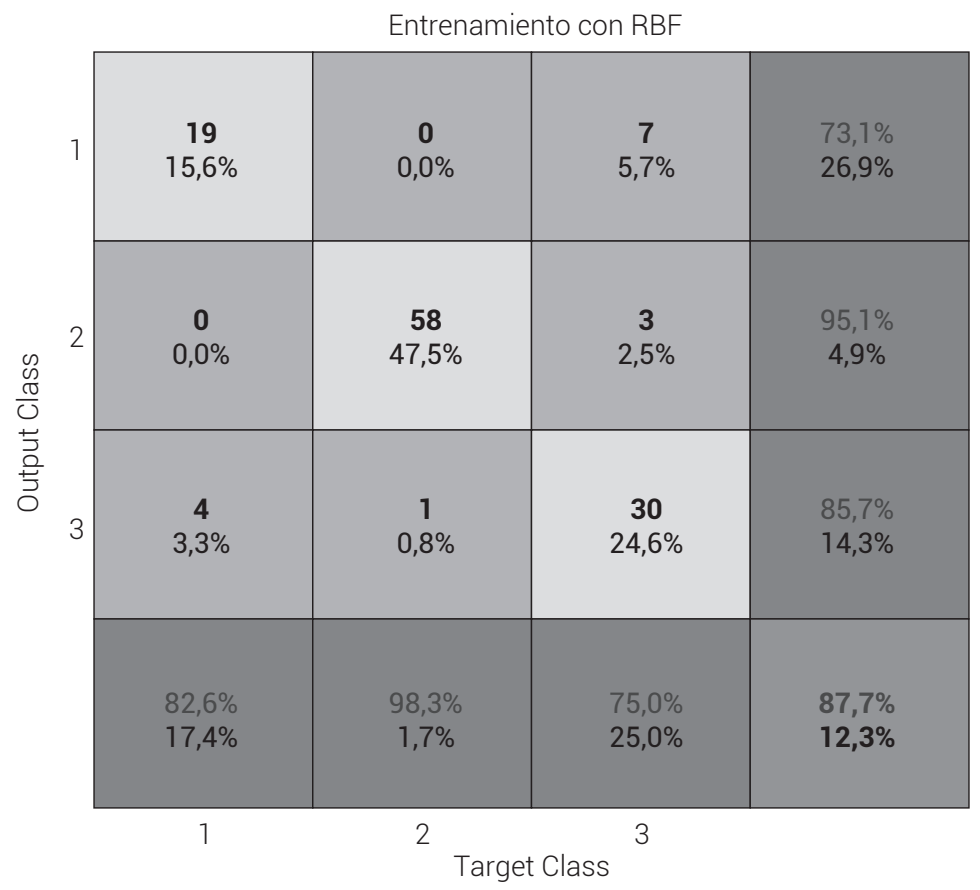

Figura 12. Resultados obtenidos con RBF

Fuente: elaboración propia

A partir de la información arrojada por las 4 figuras anteriores se puede concluir que, de los cuatro clasificadores presentados de manera individual, svm muestra el peor rendimiento muestra, sobre todo en lo que respecta a la cantidad de falsos negativos. En cambio, PNN ostenta los mejores resultados globales cuando se comparan la cantidad de falsos positivos y negativos obtenidos. Pese a que Naïve Bayes y RBF no presentan incongruencias entre patologías, el número de falsos positivos y negativos son bastante altos en comparación con PNN.

\subsection{Combinación de clasificadores}

La figura 13 muestra los resultados obtenidos para un análisis usando la combinación de los clasificadores empleados. 


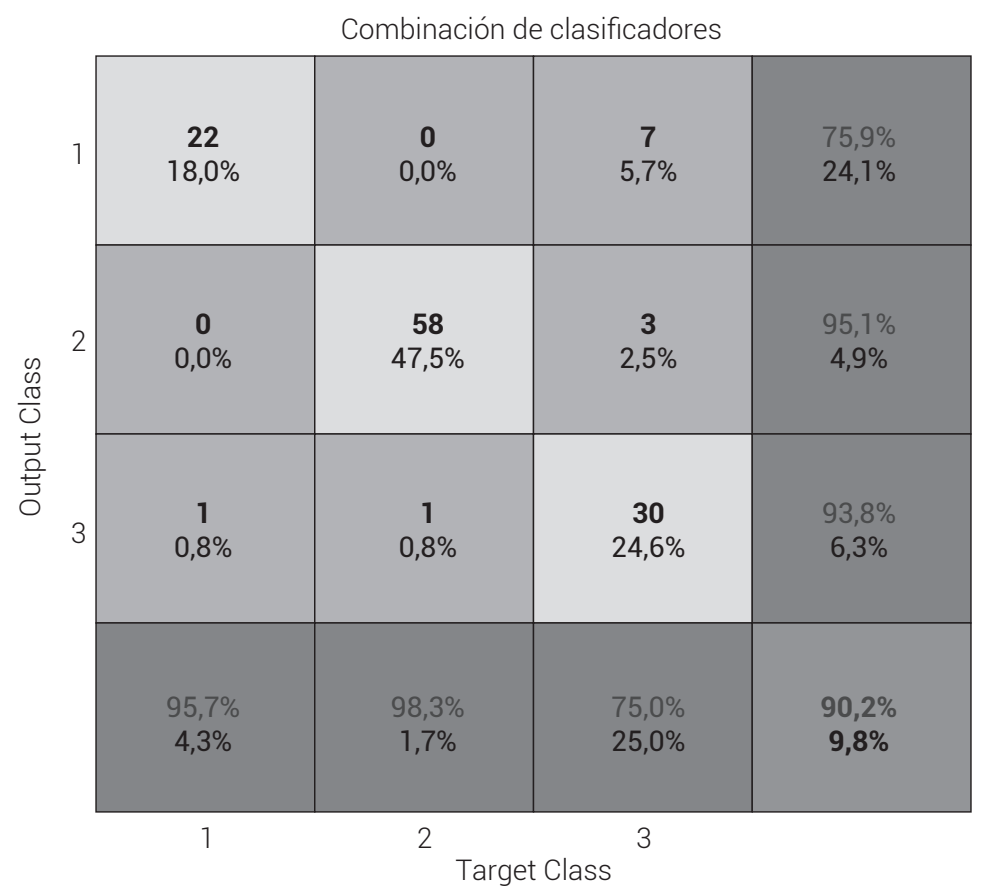

Figura 13. Resultados obtenidos con la combinación de clasificadores Fuente: elaboración propia

\section{Discusión}

El presente trabajo resalta la importancia de continuar con el desarrollo de actividades de investigación que permitan establecer el uso de máquinas de aprendizaje como parte de futuros protocolos para el estudio de patologías en Colombia, habida cuenta de que prácticas de este tipo no solo pueden aplicarse en los problemas de columna vertebral, sino en cualquier patología que pueda presentarse en el ser humano.

Además, es importante que los algoritmos empleados para realizar este tipo de diagnósticos contengan información detallada de los pacientes a tratar, así como la posibilidad de compararla con datos obtenidos de una persona sana. Lo anterior brindará la posibilidad de efectuar un diagnóstico mucho más asertivo, encaminado a prevenir que se contraiga alguna afección de forma permanente.

Una limitación presentada en el estudio de las patologías de columna vertebral tratadas durante el desarrollo de este trabajo consistió en que, si bien el conjunto de datos utilizado contiene información de personas reales, esta no brinda información completa de su columna vertebral, lo cual llevó a la aparición de errores sobre la toma de decisiones para las diferentes técnicas empleadas durante los procesos de 
clasificación. Sobre esto debe tenerse en cuenta que cada individuo ostenta características propias, las cuales que pueden suministrar información importante al realizar los procesos de clasificación; dentro de ellas se destacan las curvaturas, el sacro e incluso la longitud de la columna vertebral.

\section{Conclusiones}

Los resultados obtenidos con cada uno de los clasificadores implementados de forma independiente fueron buenos para los procesos de clasificación. Sin embargo, estos porcentajes de rendimiento no son lo suficientemente buenos para realizar diagnósticos de patologías médicas, pues existe la probabilidad de que a un individuo sano se le determine alguna patología, o viceversa. Por otra parte, se puede observar que cada una de las técnicas implementadas discrimina mejor una patología específica: la de Bayes presenta mejores resultados al clasificar las muestras de la clase 2 (48,4 \%), mientras que las otras alcanzan solo el 47,5 \% en la clasificación de muestras de la misma clase; estos resultados abren la posibilidad de que se implemente un estudio en el que se emplee un clasificador por cada una de las clases. De otra parte, si se toman en consideración los pesos por cada una de las clases al llevar a cabo el proceso de combinación de los clasificadores empleados, se podrían obtener mejores respuestas de clasificación en las patologías tratadas; ello traería consigo una mejor eficiencia en los métodos propuestos.

Al implementar el algoritmo para la combinación de los clasificadores se obtuvieron, según la matriz de confusión que se presentó en la figura 13, 2 falsos positivos y 10 falsos negativos, lo cual no presenta problemas en la clasificación entre patologías. No obstante, pese a los resultados obtenidos, no se logra cumplir con la meta de mejorar el resultado obtenido por los clasificadores implementados de forma individual, puesto que el algoritmo PNN presenta un valor porcentual global similar.

\section{Referencias}

[1] R. N. Ajalmar y A. B. Guilherme, "On the Application of Ensembles of Classifiers to the Diagnosis of Pathologies of the Vertebral Column: A Comparative Analysis”, IEEE Latin America Transactions, vol. 7, n. ${ }^{\circ}$ 4, pp. 487-496, 2009. doi: https://doi.org/10.1109/TLA.2009.5349049 


\section{Diego Fernando Ramírez-Jiménez, Julián David Quintero-Ospina}

[2] A. Rocha, R. Sousa, G. Barretoy J. Cardoso, Diagnostic of Pathology on the Vertebral Column with Embedded Reject Option. Porto: Departamento de Ingeniería de Teleinformática, Universidad Federal de Ceará (UFC), INESC Porto, Facultad de Ingeniería, Universidad de Porto, p. 1, 2011. doi: https://doi.org/10.1007/978-3-642-21257-4_73

[3] C. M. Bishop, Pattern Recognition and Machine Learning. Berlín, Alemania: Springer, pp. 20-34, 2006.

[4] R. Duda, P. Hart and D. Stork, Pattern Classification. Hoboken, Nueva Jersey: Wiley, pp. 3-24, 2001.

[5] E. Alpaydin, Introduction to Machine Learning. Cambridge, Massachusetts: The MIT Press, pp. $1-20,2010$

[6] S. J. Russell y P. Norvig, Inteligencia Artificial Un Enfoque Moderno. Madrid, España: Pearson Educación, pp. 2-5, 2004.

[7] J. Han, M. Kamber and J. Pei, Data Mining Concepts and Techniques. Waltham, Massachusetts: Morgan Kaufmann, pp. 23-32, 2012.

[8] Digital Guide, "Machine learning ien qué consiste el aprendizaje automático?", Machine Learning, 2018 [En línea]. Disponible en: https://www.1and1.es/digitalguide/online-marketing/analisis-web/machine-learning-asi-piensan-las-maquinas/

[9] I. Hidayah, A. Erna y M. A. Kristy, "Application of J48 and Bagging for Classification of Vertebral Column Pathologies", International Conference on Information Technology and Multimedia (ICIмu), pp. 314-317, 2014. doi: https://doi.org/10.1109/ICIMU.2014.7066651

[10] S. Ansari, F. Sajjad, Z. Qayyum, N. Naveed y I. Shafi, "Diagnosis of Vertebral Column Disorders Using Machine Learning Classifiers", International Conference on Information Science and Applications (ICISA), p. 1, 2013. doi: https://10.1109/ICISA.2013.6579446

[11] Y.UnalyH.E. Kocer, "Diagnosis of Pathologyon theVertebral Columnwith Backpropagationand Naive Bayes Classifier", The International Conference on Technological Advances in Electrical, Electronics and Computer Engineering (TAEECE), pp. 276-279, 2013. doi: https://10.1109/ TAEECE.2013.6557285

[12] A. Babalik, I. Babaoğlu y A. Özkış, "A Pre-Processing Approach Based on Artificial Bee Colony for Classification by Support Vector Machine", International Journal of Computer and Communication Engineering, pp. 68-70, 2013. doi: https://doi.org/10.7763/IJCCE.2013.V2.139 
[13] UCI Machine Learning Repository, "Vertebral Column Data Set", ucı Machine Learning Repository 2015 [En línea]. Disponible en: http://archive.ics.uci.edu/ml/datasets/Vertebral+Column\#.

[14] Organización Panamericana de la Salud, Oficina Sanitaria Panamericana, Clasificación estadística internacional de enfermedades y problemas relacionados con la salud. Washington: Oficina Regional de la Organización Mundial de la Salud, pp. 1, 2003.

[15] Societat Catalana d’Anestesiologia, Reanimació i Terapèutica del Dolor, "Dolor de espalda. Diagnóstico. enfoque general del tratamiento”, Societat Catalana d'Anestesiologia, Reanimació i Terapèutica del Dolor, pp. 1, 2015. [En línea]. Disponible en: http://www.scartd.org

[16] E. Gutiérrez, "Aplicación de las máquinas de soporte vectorial para el reconocimiento de matrículas", tesis, Universidad Pontificia Comillas, Escuela Técnica Superior de Ingeniería (ICAI), Madrid, pp. 1-94, 2007.

[17] E. Abdrabou, "A Hybrid Intelligent Classifier for the Diagnosis of Pathology on the Vertebral Column", Artificial Intelligence Methods and Techniques for Business and Engineering Applications, Ene., pp. 297-310, 2012.

[18] M. T. Escobedo y J. A. Salas Plata Mendoza, "P. Ch. Mahalanobis y las aplicaciones de su distancia estadística”, Culcyt Estadística, vol. 5, n. ${ }^{\circ}$ 27, pp. 13-20, 2008.

[19] Matlab ${ }^{\circledR}$, "Moutlier1: Detection of Outlier in Multivariate Samples Test", Matlab File Exchange, 2006 [En línea]. Disponible en: http://www.mathworks.com/matlabcentral/fileexchange/ loadFile.do?objectld=12252

[20] V. Otero, "reconocimiento de localizaciones mediante máquinas de soporte vectorial," Tesis, Universidad Carlos III de Madrid, Escuela Politécnica Superior, Madrid, pp. 1-96, 2012.

[21] J. Pérez Mesa, J. Valenciano y J. Levy Manguin, "Empleo de redes neuronales de base radial a un modelo econométrico de exportación de tomate," Ciencia Ergo Sum, Universidad Autónoma del Estado de México, vol. 14, no. 1, Mar., pp. 6 - 14, 2007.

[22] S. Reddy, S. Kodali y J. Lakshmi, "Classification of Vertebral Column using Naïve Bayes Technique", International Journal of Computer Applications, vol. 58, n. ㄱ, Nov., pp. 38-42, 2012.

[23] R. Q. Juan y C. M. Mario, "Redes neuronales artificiales para el procesamiento de imágenes, una revisión de la última década", RIEE\&C, Revista de Ingeniería Eléctrica, Electrónica y Computación, vol. 9, n. ${ }^{1}$, Jul., pp. 7-16, 2011. 
[24] J. A. Cadena, C. J. M. Cadena y S. Pérez Londoño, "Aplicación de redes neuronales probabilísticas en la detección de fallas incipientes en transformadores”, Scientia et Technica, año XIV, vol. 14, n. ${ }^{3}$ 39, Sep., pp. 48-53, 2008.

[25] K. Josef, H. Mohamad, D. Robert y M. Jiri, "On Combining Classifiers”, IEEE Transactions on Pattern Analysis and Machine Intelligence, vol. 20, n. ${ }^{\circ}$ 3, pp. 226-239, 1998. doi: https://doi. org/10.1109/34.667881 